\title{
Influence of Placement of Small Space Vectors on the Performance of PWM Techniques for Three Level Inverters
}

\author{
Abdul Rahiman Beig \\ Department of Electrical Engineering \\ Indian Institute of Science \\ Bangalore 560012 \\ INDIA
}

arbeig@ieee.org

V.T.Ranganathan

vtran@ee.iisc.ernet.in

\begin{abstract}
The performance of three level inverters depends on the PWM technique. The three level space vector PWM (SVPWM) has improved performance in terms of the THD and the D.C. bus mid point voltage balance compared to three level sine triangle PWM (SPWM). The objective of this paper is to study the importance of the space vectors, the influence of their placement in the switching sequence on the performance of the PWM techniques and address the basic issue of why the SVPWM has superior performance over SPWM technique. Such a basic understanding will help in designing optimal PWM with desired performance. The study has shown that the superior performance of SVPWM is because of the placement of the small space vectors as the beginning and ending vectors in each sequence, with equal dwell times. The simulation results are presented to validate the theoretical observations. The SVPWM is implemented on a general purpose DSP based digital controller and applied to constant $\nu / f$ drive.
\end{abstract}

Index Terms - Three Level Inverters, Diode Clamp Three Level inverters, Space Vector PWM (SVPWM), Sine Triangle PWM (SPWM).

\section{INTRODUCTION}

The circuit diagram of the three-level diode clamp VSI is shown in fig 1. Each pole can he connected to positive D.C. bus (state I) or D.C. bus mid point (state 0) or negative D.C. bus (state -1 ). Fig. 2 shows the space vectors of the threelevel inverter on $a-\beta$ plane. There are $2^{3}=21$ inverter

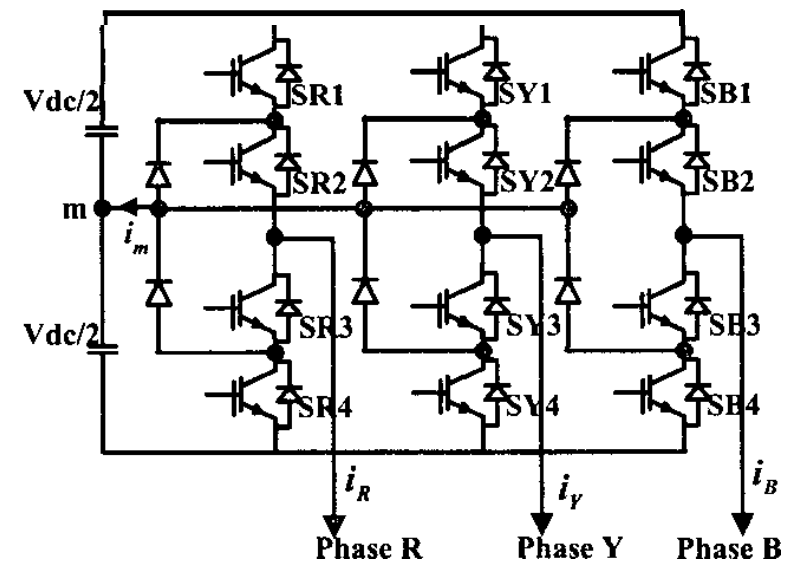

Fig. 1 Circuit digram of three level diode clamp inverter states, which can be used to produce 19 space vectors. The space vectors $\quad \bar{\gamma} 7, \bar{\gamma} 9, \bar{V} 11, \bar{V} 13, \bar{V} 15$ and $\bar{\gamma} 17$ with magnitude $V_{d c}$ form the set of six large space vectors. Similarly the set of six medium space vectors consists $\bar{V} 8, \bar{V} 10, \bar{V} 12, \bar{V} 14, \bar{V} 16$ arid $\bar{V} 18$ with magnitude $0.866 V_{d c}$. Each of the medium and large vectors can be generated by only one inverter state a!; defined in fig. 2. There are six small vectors, $\bar{V} 1$ to $\bar{V} 6$, with magnitude $0.5 V_{d c}$. Each of these small vectors can he generated by two inverter states as defined in fig. 2 and table I. The zero space vector, $\vec{y} 0$, can be generated by three inverter states namely $\left(\begin{array}{llll}0 & 0 & 0\end{array}\right)$ or $\left(\begin{array}{lll}1 & 1 & \mathrm{I}\end{array}\right)$ or $(-1-1-1)$

Several types of PWM techniques have been developed for three-level diode clamp inverters [2-16]. These modulation techniques can be grouped into two categories: sine triangle PWM (SPWM) and space vector PWM (SVPWM). In the case of three level diode clamp voltage source inverters the performance of the PWM techniques are characterized by the output current ripple, THD, D.C. bus mid point 'voltage imbalance and common mode voltage variation. A large variation of D.C. bus mid point voltage will disturb the half wave symmetry and will give rise to even harmonics. The

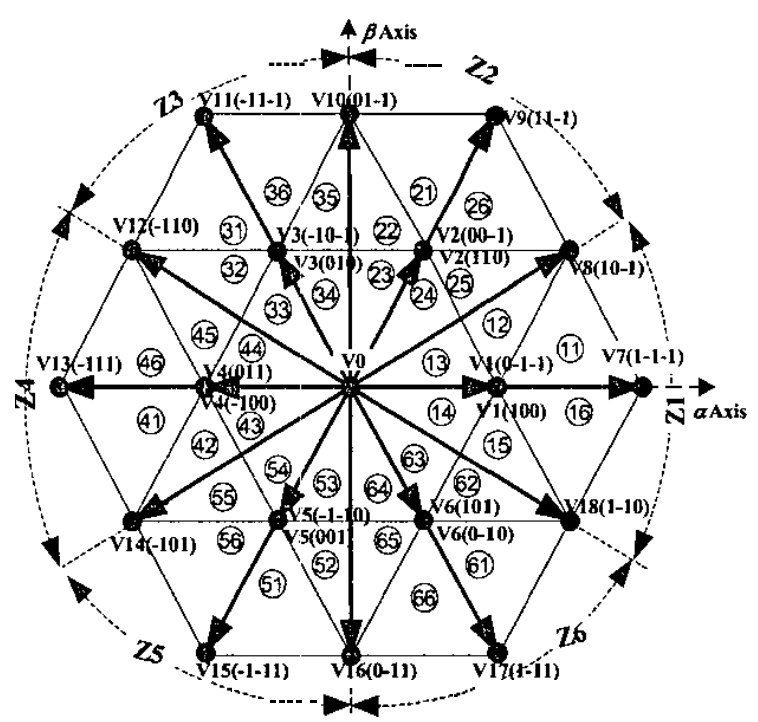

Fig. 2 Space vectors of three level diode clamp inverter 
presence of even harmonics will not only affect the THD hut also affects the performance of the drive [13]. Even though several PWM schemes are developed to address some of the specific problems like improving the THD [2-9], reducing the D.C. bus imbalance [10-14], minimizing the common mode voltage [15-17], there is still need to develop an optimal PWM algorithm that can result in desired overall performance. The objective of this paper is to study the switching space vectors of the SVPWM and SPWM techniques and establish a basic relation between the switching vectors and the performance of the PWM techniques. Such a basic understanding will help in designing the optimal PWM algorithms with desired performance.

Based on the above study it is shown that it is the proper choice of small space vectors and their placement at the beginning and end of the switching sequences with equal dwell time, which results in low voltage ripple and hence low THD. SVPWM techniques employ this type of sequence; hence their performance is superior compared to other PWM techniques in terms of $T H D$. It is also illustrated that such an arrangement will result in reduced D.C. bus mid point voltage imbalance and reduced common mode voltage variation. Thus the SVPWM sequence has superior overall performance compared to other PWM techniques in the linear range of the modulation. Another contribution of this paper is that the region in which the given small vector has improved performance is established; thereby removing the ambiguity in the choice of small vectors.

The principles of SVPWM and SPWM are reviewed in section II. In section III, the influence of the placement of space vectors on voltage ripple and flux ripple is established, The mean square value of the flux ripple of SVPWM is computed and plotted for the entire linear range of the modulation. The SVPWM and SPWM algorithms are simulated and the WTHD of the line voltages are presented. The SVPWM algorithm is applied to an experimental three level diode clamp VSI based constant $v / f$ drive. The experimental results are given in section IV.

\section{II . REVIEW OF THREE LEVEL PWM TECHNIQUES}

\section{A. SVPWMfor Three level Inverters:}

In space vector approach to PWM the reference vector $\bar{V}_{r}$ is sampled at regular intervals $T_{S}$. The sampled reference vector is approximated by time averaging the nearest three vectors $\widetilde{V}_{x}, \bar{V}_{y}$ and $\bar{V}_{z}$ according to (1)

$\bar{V}_{r} T_{s}=\bar{V}_{x} T_{x}+\bar{V}_{y} T_{y}+\bar{V}_{z} T_{z}$

where, $T_{x}, T_{y}$ and $T_{z}$ are the intervals of $\bar{V}_{x}, \bar{V}_{y}$ and $\bar{V}_{z}$ respectively and $T_{s}=T_{x}+T_{y}+T_{z}$.

In (1), for $m>0.433$, where $m$ is the modulation index, defined as the ration between the magnitude of $\bar{V}_{r}$ to the $V_{\text {dic }}$;
TABLE I

SMALL VECTORS, THEIR STATES AND D.C. BUS MID POINT CURRENT

\begin{tabular}{|c|c|c|c|c|c|}
\hline \multirow{2}{*}{$\begin{array}{c}\text { Sector } \\
Z\end{array}$} & \multirow{2}{*}{$\begin{array}{c}\text { Small } \\
\text { vector } \\
\bar{V}_{\boldsymbol{z}}\end{array}$} & \multicolumn{4}{|c|}{ Small vectors and mid point current } \\
\hline & & $\bar{V}_{z x}$ & $i_{m z x}(t)$ & $\bar{V}_{z y}$ & $i_{m z y}(t)$ \\
\hline 1 & $\bar{V}_{1}$ & 100 & $i_{R}$ & $0-1-1$ & $-i R$ \\
\hline 2 & $\overline{V_{2}}$ & 110 & $-i_{B}$ & $\begin{array}{llll}\mathbf{0} & 0 & -1\end{array}$ & $i_{B}$ \\
\hline 3 & $\overline{V_{3}}$ & 010 & $i Y$ & $-10-1$ & $-i Y$ \\
\hline 4 & $\bar{V}_{4}$ & O I I & $-i_{R}$ & -100 & $i_{R}$ \\
\hline 5 & $\bar{V}_{5}$ & $00 \mathrm{I}$ & $i_{B}$ & $-1-10$ & $-i_{B}$ \\
\hline 6 & $\overline{V_{6}}$ & 100 & $-i_{Y}$ & $0-1 \mathbf{0}$ & $i y$ \\
\hline
\end{tabular}

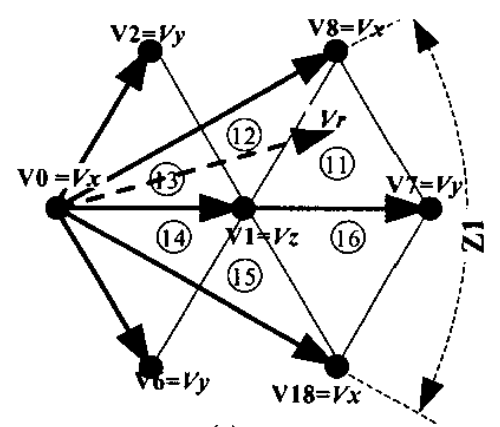

(a)

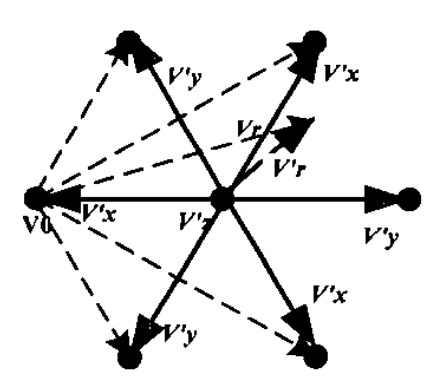

(b)

Fig.3.(a) Space vectors associated with sector 1 .

(b) Mapping of the vectors of sector 1 to fictitious vectors.

all the nearest three vectors are non zero vectors and there is no common vector in all the regions of the space phasor. "The solution of (1), can he simplified by exploring the symmetry in the space vector plane. The entire space vector plane is divided in to six symmetrical sectors, each of sixty degrees interval as shown in fig.2. Each sector $Z$, where $Z=1,2, \ldots 6$, is associated with one small vector $\bar{\gamma}_{z}$ at the center and six other vectors at the vertices of the hexagon. These small vectors are defined in table $I$. The small vector $\vec{V}_{1}$ and other six vectors of sector 1 are redrawn in fig. 3 (a). The vectors of the other sectors are phase displaced by $(Z-1) \pi / 3$ radians. All the vectors of a given sector can be mapped to seven fictitious vectors, with small vector as the origin according to (2). 


$$
\begin{aligned}
& \bar{V}_{r}^{\prime}=\bar{V}_{r} e^{j(Z-1) \pi / 3}-\bar{V}_{1} \\
& \bar{V}_{x}=\bar{V}_{x} e^{j(Z-1) \pi / 3}-\bar{V}_{1} \\
& \overline{\bar{V}}_{y}^{\prime}=\bar{V}_{y} e^{j(Z-1) \pi / 3}-\bar{V}_{1} \text { and } \\
& \bar{V}_{z}^{\prime}=\bar{V}_{z} e^{j(Z-1) \pi / 3}-\bar{V}_{1}=0
\end{aligned}
$$

The mapping of the all the seven vectors of sector in to seven fictitious vectors is illustrated in fig. 3(b). The magnitude of $\vec{V}_{z}^{\prime}$ is always zero and (1) will have only two unknowns as in (3).

$$
\bar{V}_{r}^{\prime} T_{s}=\bar{V}_{x}^{\prime} T_{x}+\bar{V}_{y}^{\prime} T_{y} \text { and } T_{z}=T_{s}-T_{x}-T_{y}
$$

The $T_{x}, T_{y}$ and $T_{z}$ are computed using (3).

The samples will have sequences $\bar{V}_{z x} \rightarrow \bar{V}_{x} \rightarrow \bar{V}_{y} \rightarrow \bar{V}_{z y}$ and $\bar{V}_{z x} \leftarrow \bar{V}_{x} \leftarrow \bar{V}_{y} \leftarrow \bar{V}_{z y}$ alternatively, with the dwell times $T_{z x}, T_{x}, T_{y}$ and $T_{z y}$ respectively, where $T_{z x}=T_{z y}=T_{z} / 2$. Unlike two level inverters, the $\bar{V}_{z}$ vector will change from sector to sector. So there will be one additional switching during sector change over. These SVPWM sequences will guaranty that only one switch will switch during state transition.

\section{B. SPWM for Three level Inverters:}

There are different types of SPWM algorithms. The most popular type of SPWM employs two carrier signals. The carrier signals may be in phase or out of phase [3]. The SPWM employing two carrier signals, which are in phase is shown in fig. 4. The switching sequences are similar to that of SVPWM but the dwell time $T_{z}$ of the small vectors is unequally divided between $\bar{V}_{z x}$ and $\bar{V}_{z y}$, space vectors such that $T_{z x}+T_{z y}=T_{z}[5-7]$. The sequences guaranty that only one switch will change status during state transition.

\section{SIGNIFICANCE OF PLACEMENT OF SMALL VECTORS ON THE PERFORMANCE OF PWM TECHNIQUES}

\section{A. Effect of Placement of Small Vector on THD}

The difference between the inverter output voltage in the $j^{\text {th }}$ state of the switching sequence $\left(\bar{V}_{j}\right)$ and the reference voltage $\left(\bar{V}_{r}\right)$ will result in voltage ripple as defined in (4).

$$
\Delta \bar{V}=\bar{V}_{j}-\bar{V}_{r}
$$

The voltage ripple causes the flux ripple in the machine: which is responsible for the current ripple and harmonics.

The magnitude of the flux ripple $\Delta \psi$, can be easily computed by resolving the voltage ripple in to alpha and beta components as in (5).

$$
\begin{aligned}
& \Delta \psi_{\alpha}=\int \Delta V_{\alpha} d t=\int\left(V_{j \alpha}-V_{r \alpha}\right) d t \quad \text { and } \\
& \Delta \psi_{\beta}=\int \Delta V_{\beta} d t==\int\left(V_{j \beta}-V_{r \beta}\right) d t
\end{aligned}
$$

The mean square value of the flux ripple over a sector, defined in (6) can be taken as a measure of THD to study the
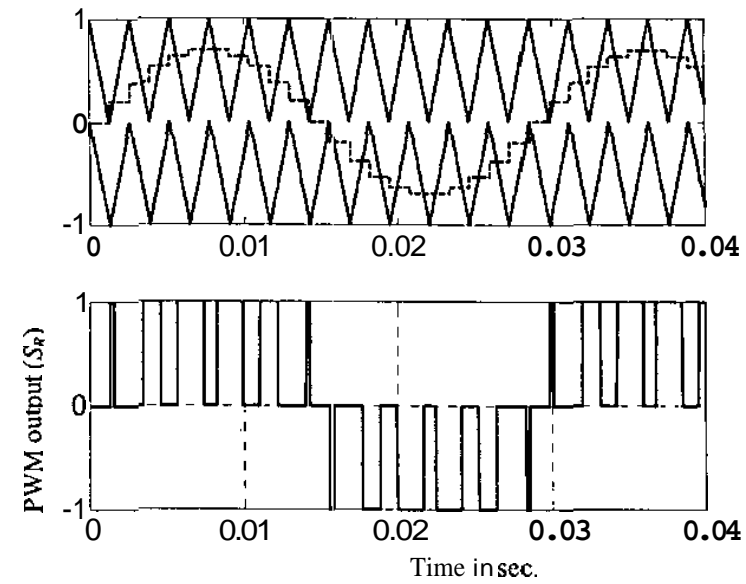

Fig. 4. SPWM: Carrier and modulating signals and PWM output performance of the PWM techniques $[2,3]$

$$
\Delta \psi^{2}=\frac{6}{N} \sum_{k=1}^{N} \Delta \psi_{k}^{2}
$$

where $N$ is the number of samples per sector and $\Delta \psi_{k}^{2}$ is the square of the magnitude of the flux ripple of the $k^{\text {th }}$ sampling interval computed over one sampling interval $\left(T_{s}\right)$, using $\Delta \psi_{k}^{2}=\Delta \psi_{k \alpha}^{2}+\Delta \psi_{k \beta}$. The aandpcomponents of the $\Delta \psi_{k}^{2}$ over one sampling interval are computed as follows.

$$
\begin{aligned}
\Delta \psi_{k \alpha}^{2}= & \frac{1}{T_{s}}\left[\int_{0}^{T z x}\left(V_{z x \alpha}-V_{r \alpha}\right)^{2} d t+\int_{T z x}^{T z x+T x}\left(V_{x \alpha}-V_{r \alpha}\right)^{2} d t+\right] \\
+ & {\left[\int_{T z x+T x}^{T z x+T x+T y}\left(V_{y \alpha}-V_{r \alpha}\right)^{2} d t+\int_{T z x+T x+T y}^{T s}\left(V_{z y \alpha}-V_{r \alpha}\right)^{2} d t\right] } \\
\Delta \psi_{k \beta}^{2}= & \frac{1}{T_{s}}\left[\int_{0}^{T z x}\left(V_{z x \beta}-V_{r \beta}\right)^{2} d t+\int_{T z x}^{T z x+T x}\left(V_{x \beta}-V_{r \beta}\right)^{2} d t+\right] \\
& +\left[\int_{T z x+T x}^{T z x+T x+T y}\left(V_{y \beta}-V_{r \beta}\right)^{2} d t+\int_{T z x+T x+T y}^{T s}\left(V_{z y \beta}-V_{r \beta}\right)^{2} d t\right]
\end{aligned}
$$

Fig. 5 and 6 show the variation of alpha and beta components of the voltage ripple and flux ripple over one sampling interval (with $T s=1.7$ millisecond., $m=0.4, k=4$ and $N=5$ ) for three different combinations of placement of small vectors. Case (a) CSVPWM sequences: The dwell time $T_{z}$ is equally divided between $\bar{V}_{z x}$ and $\bar{V}_{z y}$ (Fig. 5a and 6a).

Case (b) SPWM: The dwell tine $T_{z}$ is unequally divided between $\bar{V}_{z x}$ and $\vec{V}_{z y}$, such that $T_{z x} \neq T_{z y}$ (Fig. 5b and 6b).

Case (c) Clamping or discontinuous PWM sequences: No division of the dwell time $T_{z}$. The small vector is placed either at the beginning or at the end (Fig. $5 \mathrm{c}$ and $6 \mathrm{c}$ ).

The flux ripple and the mean square value of the flux ripple are low for case (a) and are high for case (c). Their values are in between for case (b). From the above analysis following observations can be drawn. 
- The voltage ripple, hence the flux ripple is a function of the dwell time of the space vectors and their placement in the switching sequence. If the space vector is close to the reference vector the dwell time of the space vector will be high and the voltage ripple will be low.

- For the sector definition of fig. 2, for a given sector, the
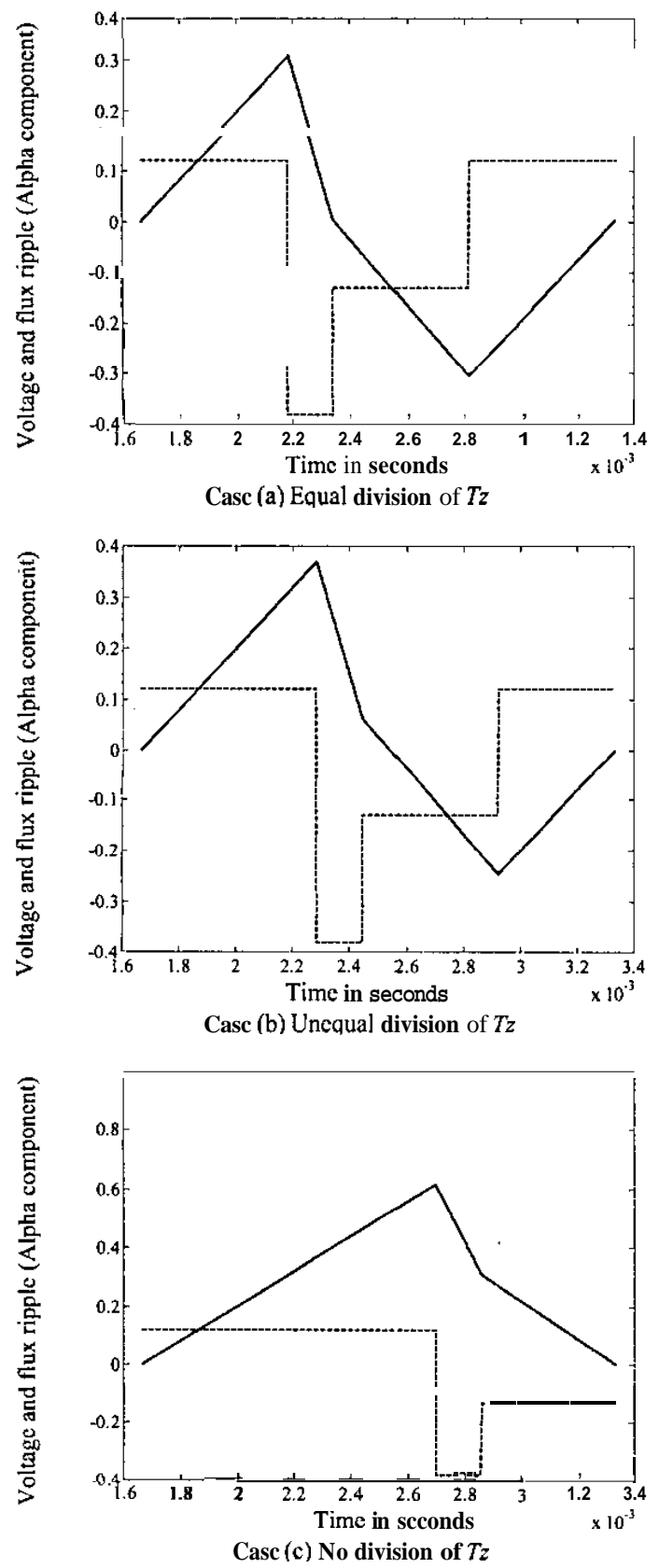

Fig. 5. The Variation of alpha component of voltage ripplc ( --1$)$ and flux ripplc ( $\longrightarrow$ over one smapling interval. ( $T s=1.7 \mathrm{msec}, m=0.4, k=4$ and $N=5$ ) (a) Equal Division of Tz interval (b) Uncqual division of $T z$ intcrval $T z x>T z y$ (c) No division of $T z$ interval (The flux ripple is magnified by a factor of $\$ 000$ ) small vector associated with the sector will be the nearest space vector for all the samples in the region $0.25 \leq m \leq 0.75$. Hence for $0.25 \leq m \leq 0.75, T_{z}$ is greater than $T_{X}$ and $T_{y}$. In SVPWM $T_{z}$ is equally split into two halves between $\bar{V}_{z x}$ and $\bar{V}_{z y}$, whereas in the case of SPWM the splitting of $T_{z}$ is
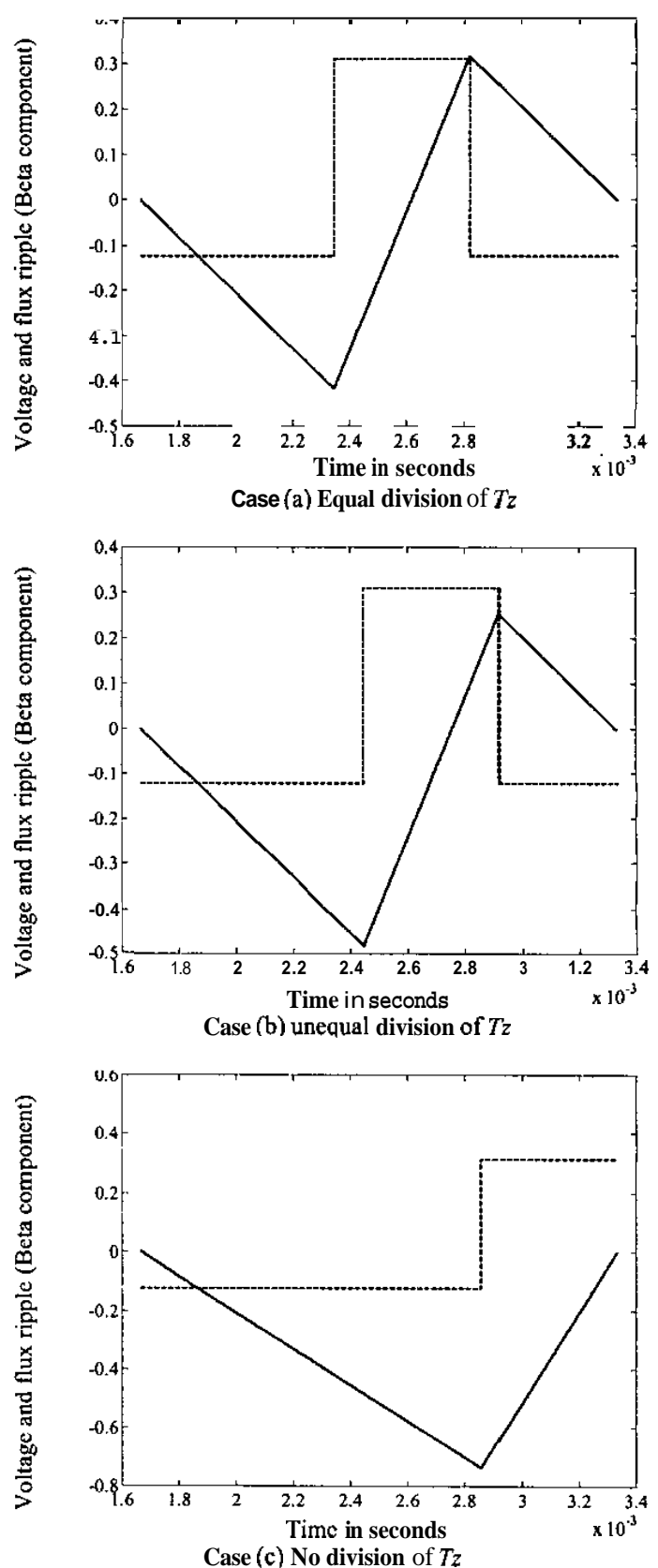

Fig. 6. The Variation of beta component of voltage ripple $(--)$ ) and flux ripplc $(\longrightarrow$ over one smapling interval. $(T s=1.7$ msec, $m=0.4, k=4$ and $N=5)$ (a) Equal Division of $\mathrm{T} z$ interval (b) Unequal division of Tz interval Tzx $>\mathrm{Tzy}$ (c) No division of Tz interval (The flux ripple is magnificd by a factor of 5000) 


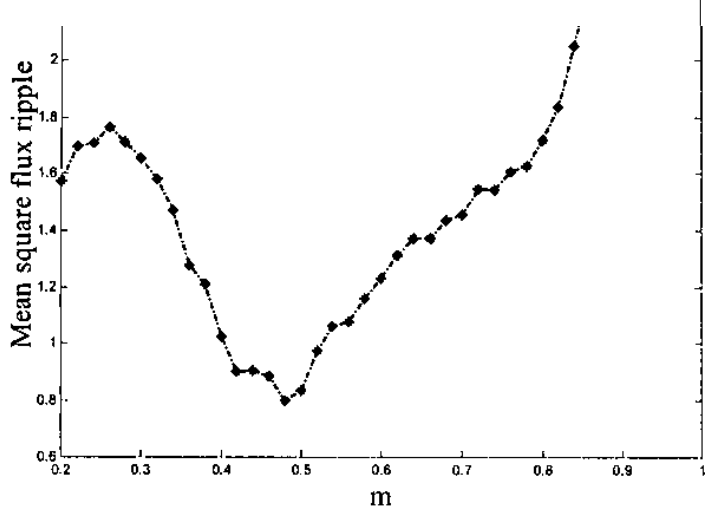

Fig. 7. Mean square flux ripple $v / \mathrm{s} \mathrm{m}$

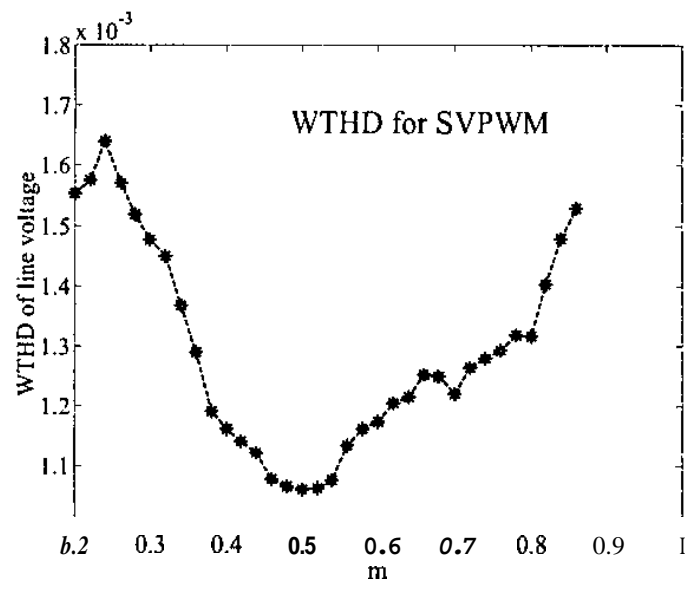

Fig.8. WTHD v/s $\mathrm{m}$ for SVPWM

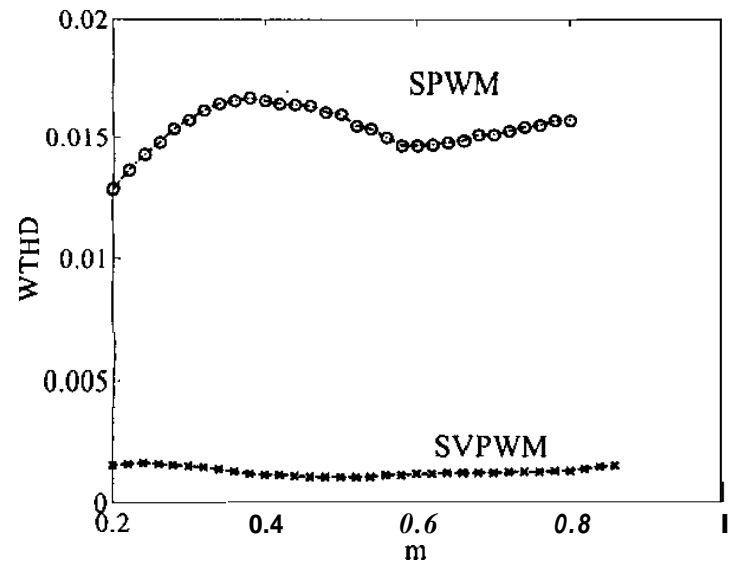

Fig. $9 \mathrm{WTHD} v / \mathrm{s} \mathrm{m}$ for SVPWM and S P W $\mathrm{M}$

unequal. This is the reason why the THD is high in the case of SPWM compared to SVPWM.

- As $m$ is increased beyond 0.75 , the $T_{x}$ or $T_{y}$ will be larger than $T_{z}$. Hence there is little or no influence of splitting the dwell time of small vectors. So the voltage ripple, the flux ripple and the $T H D$ will increase at higher values of $\boldsymbol{m}$ especially at over modulation region. Also for a given switching frequency, the sampling frequency of clamping sequences will be 1.5 times higher than that of conventional PWM schemes. This is the reason why in over modulation region, for a given switching frequency $(f s w)$, the THD of clamping sequences will be comparable or better, compared to that of'conventional PWM algorithms.

In the case of SVPWM, the mean value of the square of the flux is computed from the simulation results for the linear range of $\boldsymbol{m}$. For $f s w=2.5 \mathrm{KHz}$, the variation of mean square value of the flux ripple in the linear range of $\boldsymbol{m}$ is plotted in fig.7. As expected the mean square value of the flux ripple is low at and around $m=0.5$ and rises as one moves away from $m=0.5$ in both the directions. This is in agreement with the theoretical observations given above. The SVPWM and SPWM algorithms are simulated using MATLAB-SIMULINK toolbox. The WTHD of the line voltage is computed from the simulation results. WTHD is proportional to the $T H D$ of the motor no load current and can be taken as a measure of performance [8]. The variation of WTHD in the linear range of $\boldsymbol{m}$ is shown in Fig. 8. From fig.7 and $X$, It can be seen that WTHD varies similar to the mean square value of flux ripple and is low at and around $m=0.5$ and increases as $\boldsymbol{m}$ is either increased or decreased. So the mean square value of flux can also be taken as the index of the THD, similar to WTHD. Comparative results of WTHD of SPWM and SVPWM are shown in fig.9, and the SVPWM has improved WTHD compared to that of SPWM.

\section{B. Effect of Placement of Small Vector on D.C. Bus Mid Point Voltage}

In three level diode clamp inverters, the D.C. bus capacitors carry the load current. The unequal loading of the upper and lower capacitors causes the mid point voltage to fluctuate. The D.C. bus rnid point voltage variation $\left(\Delta V_{m}\right)$ can be estimated from the D.C. mid point bus current $\left(i_{m}\right)$ using (7)

$$
\begin{aligned}
& \Delta V_{m}=\frac{\mathrm{I}}{C} \int_{0}^{T s} i_{m}(t) d t \\
& =\frac{1}{C}\left[\begin{array}{l}
\int_{0}^{T z x} i_{m z x}(t) d t+\frac{1}{C} \int_{T z x}^{(T z z+T x)} i_{m x}(t) d t+ \\
+\int_{(T z x+T x)}^{(T z x+T x+T y)} i_{m y}(t) d t+\int_{(T z x+T x+T y)}^{T s} i_{m z y}(t) d t
\end{array}\right]
\end{aligned}
$$

The small vectors can be used to reduce $\Delta V_{m}$ and this property is extensively used to design SVPWM techniques to minimize the D.C. bus imbalance $[10-14]$. The $i_{n}$ due to the two small vectors will be equal and opposite that is $i_{m z x}(t)=-i_{m z y}(t)$ as given in table I. In the case of SVPWM switching sequences, the dwell time of $\bar{V}_{z x}$ and $\bar{V}_{z y}$ states will be equal. The $\Delta V_{m}$ due to these states is perfectly cancelled in each sampling interval, so they do not contribute for the D.C. bus capacitor voltage imbalance. In SPWM, the 
dwell time of $\bar{V}_{z x}$ and $\bar{V}_{z y}$ will he different, so the $\Delta V_{m}$ due to these states will be non-zero and there will be D.C. bus imbalance. in the case of clamping sequences, only one of the states of $\bar{V}_{z}$ is used, so there will be large imbalance in D.C. bus mid point voltage due to small vectors. Thus, the placement of small vectors at the beginning and end state with equal dwell time helps in reducing the D.C. bus imbalance. As far as $\bar{V}_{x}$ and $\bar{V}_{y}$ states are concerned, if these states fall in the set of medium or small vectors, then they contribute for the D.C. bus imbalance. The mid point current due to zero and large vectors will be zero, so they do not contribute for the D.C. bus imbalance. Under balanced three phase conditions, as in drives, the D.C. bus balance can be achieved over a cycle of the fundamental if the PWM sequences have half wave symmetry. If these sequences have three-phase symmetry also, then the D.C. bus can be

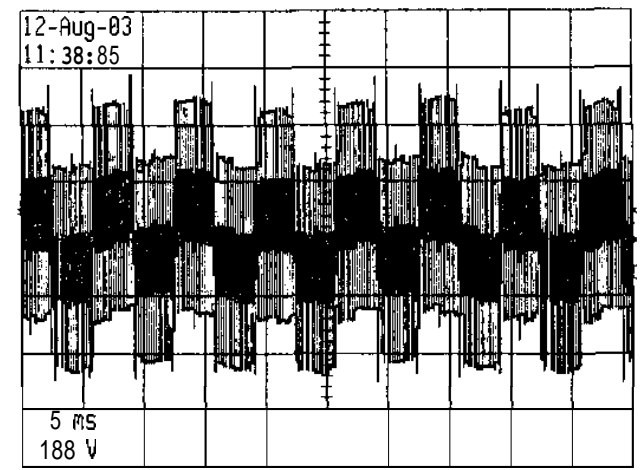

Fig 10 Experimental waveform of $v_{\pi m}$ for S VP WM with $F_{S}=50 \mathrm{~Hz}, V_{d c}=570 \mathrm{~V}$ and $f_{s} w=2.5 \mathrm{KHz}$.
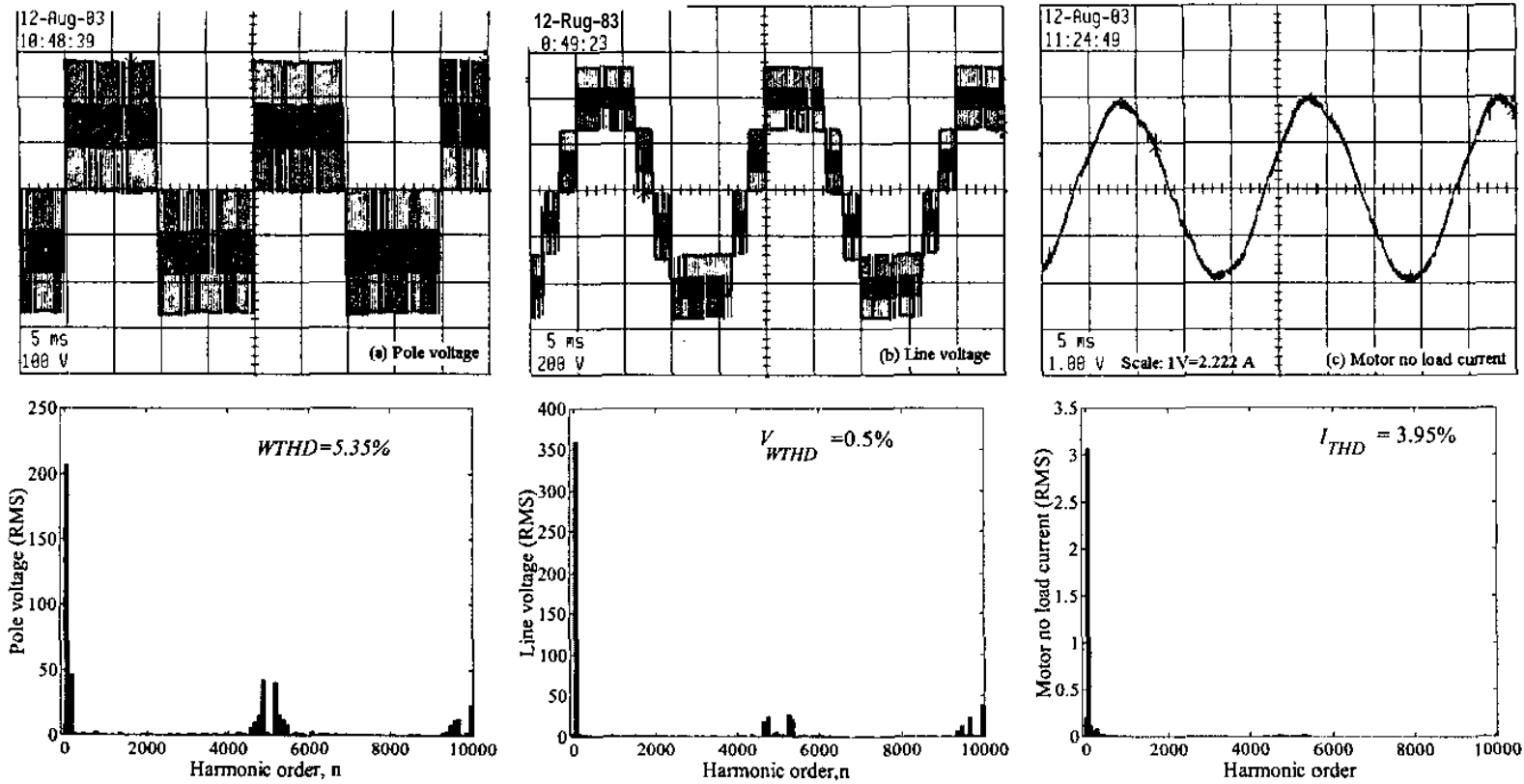

Fig. 11. Experimental results of SVPWM $\left(F s=50 \mathrm{~Hz}, V_{d c}=570 \mathrm{~V}, f s w=2.5 \mathrm{KHz}\right)$ : Pole voltage, Line voltage, Motor no load current and their harmonic spectra balanced over every $1 / 3^{\text {td }}$ period of the fundamental cycle. Compared to SPWM, SVPWM approach gives better flexibility in getting three phase and half wave symmetry, one such SVPWM is given in [8]. These sequences are generally employed for low switching frequency applications.

\section{Effect of Placement of Small Vector on Common Mode Voltage}

The common mode voltage variation, $v_{n g}$ is given by $v_{n g}=v_{n m}+v_{m g}$, where $\mathrm{n}, \mathrm{m}$ and $\mathrm{g}$ are the motor stator neutral, D.C. bus mid point and system ground points respectively. So the common mode voltage depends on the voltage $v_{n m \text {. }}$ which is a function of space vector as defined by $V n m=\frac{V_{d c}}{6}\left(S_{R}+S_{Y}+S_{B}\right)$, where $\left(S_{R}, S_{Y}, S_{B}\right)$ is the inverter state. The zero space vectors $\mathrm{VO}(111)$ and $\mathrm{VO}(-1-1-1)$ will have highest value of $v_{n m}=V_{d c} / 2$. The sequences which use small vectors at the beginning state and ending state in the switching sequence of the sampling interval, will always ensure that, only one of the switches are switched during state transition and for $m<0.433$ only $\bar{V}_{0}(000)$ vector is used. Thus the zero vectors $\bar{V}_{0}(111)$ and $\bar{V}_{0}(-1-1-1)$ are avoided. The SVPWM and SPWM, both satisfy these conditions. But the clamping sequences will result in higher common mode voltages as the clamping sequences use these zero states [9]. The experimental waveform of $V_{n m}$ for SVPWM is given in fig 10 . It should be noted that the change in common mode voltage is limited to $(1 / 6) V_{d c}$ and peak is limited to $(1 / 3) V_{d c}$. 


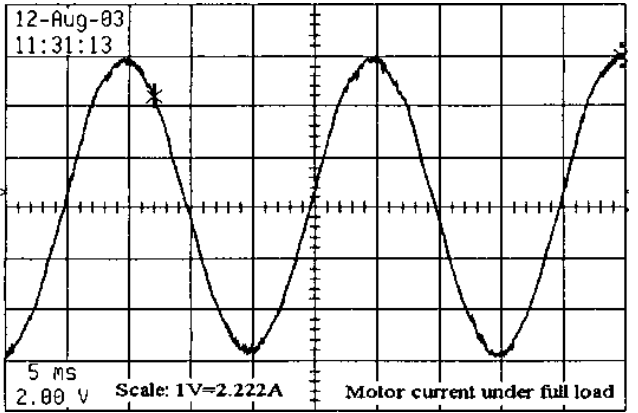

Fig. 12. Experimental results of SVPWM $(F s=50 \mathrm{~Hz}$, $V_{d c}=570 \mathrm{~V}, f_{s} w=2.5 \mathrm{KHz}$ ): Motor current at full load

\section{EXPERIMENTAL IMPLEMENTATION}

The SVPWM is implemented experimentally on TMS320 F240 DSP based digital controller. From the sampled reference vector the sector and sub-sectors are decided according to the algorithm given in section 11. The dwell time $T x, T y$ and $T z$ of nearest three vectors are calculated using (3) For a given sector and sub-sector the six PWM outputs of full compare unit of the event manager module (EVM) are programmed independently to generate the required PWM sequence. These six outputs are used to generate gate signals for the upper six devices of the inverter. These outputs are complemented externally to generate gate signals for the lower six devices. The necessary dead times are generated externally. The SVPWM is applied to constant $v / f$ drive consisting of $415 \mathrm{~V}, 50 \mathrm{~Hz}, 3$ phase, $3 \mathrm{KW}, 8 \mathrm{~A}, 1425 \mathrm{rpm}$, induction motor powered from three level IGBT based diode clamp inverter with D.C. bus voltage set to 570 volts. The typical experimental waveforms of phase voltage, line voltage and motor no lad current and their corresponding harmonic spectra at fundamental frequency $F s=50 \mathrm{~Hz}$, $V_{d c}=570 \mathrm{~V}, f s w=2.5 \mathrm{KHz}$, are given in fig. $1 \mathrm{l}$. The $T H D$ of the motor no load current gives a better measure of harmonic currents. As the load current increases, the fundamental component will increase as a result the current waveform will improve. The motor current under full load is shown in fig. 12. The THD of motor current under no load and full load conditions, computed from the experimental waveforms is $\mathbf{3 . 9 5} \%$ and $1.46 \%$ respectively.

\section{CONClusions}

The switching sequences of SVPWM and SPWM are analyzed. It is shown that even though the switching sequences of both the techniques are same, it is the placement of small space vectors as the beginning and ending state in each sequence, with equal dwell time, makes SVPWM superior compared to SPWM in terms of $\boldsymbol{T H D}$, current ripple and D.C. bus voltage imbalance. The mean square value of the flux ripple for SVPWM is plotted over entire linear range of $\boldsymbol{m}$ and it shows that the flux ripple and hence the THD will be low for $m=0.5$ and will increase as $m$ is increased or reduced. Also it can be concluded that the placement of small vector has not much influence on $\mathbf{T H D}$ at over modulation region. So in the over modulation region, for a given switching frequency, the clamping sequences will have better THD than the conventional PWM techniques.

\section{REFERENCES}

[1] Donald Grahame Holmes," The significance of zero space vector placement for carier-based PWM schemes," IEEE Tram. Industry Applaications, "vol.32, no.5, pp. 1122-1129, Sept./Oct. 1996

[2] Sandor Halasz and Alcxic Zakharoy, "Two-phase modulation technique - for three-level inverter-fed AC drives," IEEE Trans. Industrial Electronics, vol, 47,no.6, pp. 1200-1211, Dec, 2000.

[3] G.Carrara, S.Gardella, M.Marchesoni, R.Salutari and G.Sciutto, "A new multilevel PWM ncthod: A Theoretical analysis", IEEE Transaction Power Electronics, vol.7, no.5, pp 497.50, July 1992.

[4] Sandor Halasz and Alexie Zakharov "PWM strategics of three -level inverter fed AC drives" in Conf. Rec, $37^{\text {th }}$ IEEE LAS 2002, vol.2, pp 1982-1987.

[5] Fci Wang,"Sine Triangle versus space vector modulation far three-level PWM voltage-source inverters," IEEE Trans. Industry Applications, vol.38, no.2, pp500-506, Mòrch/April 2002.

[6] Yo-han Lee, Dong-Hyun Kim and Dong-Seok Hyun, "Carrier based SVPWM method for multi level system with reduced HDF," in Conf. Rec. IEEE IAS 2000, vol.3, pp, 1996-2003.

[7] Dong-Hyun Kim, Dae - W'ook Kang, Yo-han Lee and Dong-Seok Hyun, "The analysis and comparison of carricr-based PWM nethods for three level inverter," in Conf. Rec. IEEE IECON,2000, wol.2, pp. $1316 \cdot 1321$

[8] A.R.Bcig,G,Narayanan and V.T. Ranganathan, "Space Vector Based Synchronized PWM Algorithm for Three Level Voltage Source Inverters: Principles and Application to Ylf Drives,", in Proceedings of the IEEE IECON 02 conference, pp 1249-1254.

[9] A.R.Beig, and V.T, Rangarathan, "Spacc Vector Based Bus Clamped PWM Algorithms for Three Level Inverters: Implementation, Pcrformancc Analysis and Application Considerations, in Conf. Rec. of IEEE APEC 2003, pp $550-556$.

[10] Yamanaka, K., Hava, A.M.: Kirino, H,: Tanaka, Y, ;Koga, N. and Kume, T;, "A novel neutral point potential stabilization technique using the information of output current polaritics and voltage vector," IEEE Trans. Inchustry Application, vol.38, no 6, ppl572-1580, Nov/Dec. 2002.

[I I] Miao Botao; Lifu Congwei; Zhang Yang; Li Fahai;, " New SVPWM control scheme for three-phase diode clamping multilovel inverter with balanced DC voltages," in $28^{\text {th }}$ Ammual Con. Rec. IEEE IECON 2002 , vol.2, pp. 903-907.

[12] Celanovic, N.; Boroyevich, D, "A comprehensive siudy of neutral-point voltage balancing problem in three-level ncutral-point-clamped voltage source PWM inverters," IEEE Trans. Industrial Electronics, vol.15, no.2, pp 242-249, March 2000.

[13] Hyo L. Liu. Nam S Choi and Gyu H Cho, "DSP based space vector PWM for three level inverter with DC-link voltage balancing "in Con. Rec. IEEE IECON 1991, vol.2, pp. 197-203.

[14] Jac Hyeong Sco; Chang Ho Choi, "Compensation for the neutral-point potential variation in three-level spaec vector PWM in Conf. Rec., IEEE APEC Conf. 200I, Vol.2, pp. $1135-1140$.

[15] Hee-Jung Kim; Hycoun-Dong Lee: Seung-Ki Sul, "A new PWM strategy far common mode voltage reduction in neutral-point clamped inverter-fed AC motor drivas," IEEE Trans. on Industry Applications, vol37, no.6, pp 1840-1845, Nov/Dec. 2001.

[16] Haoran Zhang; von Jouanne, A,; Wallace, A., "Multilevel inverter modulation schemes to eliminate common-mode voltages," IEEE Trans. on Industy Applications. vol.36, nо.6, pp 1645-1653, Now/Dec. 2000.

[17] D. A Rendusara, Cengelce, Prasad N. Enjeti, V.R. Stefanovic and J. W Gray, "Analysis of common mode voltage -"rutettal shift" in medium voltage PWM adjustable speed drive system," IEEE Trans. Power Electronics, vol.15 No.6, pp1124-1133, Nov. 2000. 